\title{
SPectrometer for Internal Conversion Electrons (SPICE) at TRIUMF-ISAC
}

\author{
J. Smallcombe ${ }^{1, a}$, M. Moukaddam ${ }^{1}$, L. J. Evitts ${ }^{1,4}$, A. B. Garnsworthy ${ }^{1}$, S. Hallam ${ }^{1,4}$, C. Andreoiu ${ }^{3}$, G. C. Ball ${ }^{1}$, \\ C. Bolton ${ }^{1}$, R. Caballero-Folch ${ }^{1}$, M. Constable ${ }^{1}$, D. S. Cross ${ }^{3}$, P. E. Garrett ${ }^{2}$, G. Hackman ${ }^{1}$, J. Henderson ${ }^{1}$, \\ R. Henderson ${ }^{1}$, S. Ketelhut ${ }^{1}$, R. Kruecken ${ }^{1}$, L. Kurchaninov ${ }^{1}$, J. Park ${ }^{1,6}$, J. L. Pore ${ }^{3}$, E. T. Rand ${ }^{2}$, P. Ruotsalainen ${ }^{1}$, \\ J. K. Smith ${ }^{1}$, C. E. Svensson ${ }^{2}$, and M. Williams ${ }^{1,5}$
}

${ }^{1}$ TRIUMF, 4004 Wesbrook Mall, Vancouver, B.C., V6T 2A3, Canada

${ }^{2}$ Department of Physics, University of Guelph, Guelph, Ontario, Canada, N1G 2W1

${ }^{3}$ Department of Chemistry, Simon Fraser University, Burnaby, British Columbia, Canada, V5A $1 S 6$

${ }^{4}$ Department of Physics, University of Surrey, Guildford, Surrey, United Kingdom, GU2 7XH

${ }^{5}$ Department of Physics, University of York, Heslington, York, United Kingdom, YO10 5DD

${ }^{6}$ Department of Physics and Astronomy, University of British Columbia, Vancouver, British Columbia, Canada, V6T $1 Z 1$

\begin{abstract}
A new ancillary detector, SPICE (SPectrometer for Internal Conversion Electrons) has been constructed and recently commissioned for use with radioactive ion beams at the TRIUMF-ISAC II facility. SPICE is designed to be operated in conjunction with the TIGRESS High-Purity Germanium (HPGe) spectrometer to perform combined in-beam $\gamma$-ray and internal-conversion-electron spectroscopy. The main feature of SPICE is high efficiency over a wide range of electron energies from 100 to $3500 \mathrm{keV}$, with an effective reduction of beam-induced backgrounds. SPICE will be a powerful tool to measure conversion coefficients and $E 0$ transitions in atomic nuclei. A recent in-beam commissioning experiment demonstrates the effectiveness of the basic design concept of SPICE in background suppression.
\end{abstract}

\section{Introduction}

In spherical nuclei, near closed shells, many excitations can be well explained by single-particle excitations in the microscopic picture of the nuclear shell model [1]. When attempting to model deformed nuclei, one must often rely on macroscopic collective models to explain the observed lowest energy excitations [2]. Typically the transition of nuclear structure between these two regimes occurs gradually with the addition of many valance nucleons, but in some special cases this transition is observed to occur with the addition of just a couple of pairs of valence nucleons. A key area of research is to understand the microscopic mechanisms responsible for the onset of deformation, and to understand the interplay between collective and singleparticle excitations in deformed nuclei. This is particularly true when trying to explain the structure of nuclei identified as displaying shape coexistence at low excitation energy [3].

Low-lying $0^{+}$states with different deformation to the ground state are a primary feature of shape coexistence. Hence, understanding the nature of low-lying $0^{+}$states in deformed nuclei, and the excitations built upon them, is of particular interest. This can be experimentally challenging for several reasons: firstly the branching ratio for intraband $E 2$ transitions in bands built upon such excited $0^{+}$ states are much smaller than the inter-band transitions de- exciting to the ground-state band, simply because of the larger transition energy (even when the intra-band $B(E 2)$ value is much larger), secondly all the experimental observables, such as energy level spacing, branching ratios and reduced transition strengths, can be modified by band mixing with other configurations. This frequently obscures the true nature of $0^{+}$band-head states and confuses the assignment of structures built upon them.

SPICE (SPectrometer for Internal Conversion Electrons), at TRIUMF-ISAC, is a recently commissioned device used to perform in-beam electron spectroscopy with stable and radioactive ion beams. The spectrometer will be used to measure electric monopole transitions in exotic nuclei. Electric monopole transition strengths, $\rho^{2}(E 0)$, are crucial observables in the interpretation of nuclear structure. The electric monopole strength between two states of the same spin and parity is directly proportional to the difference in their mean-squared charge radii and the degree of mixing between the two configurations. SPICE will enable the identification of excited $0^{+}$states in exotic nuclei and the determination of $\rho^{2}(E 0)$ values, which will help develop our understanding of shape coexistence away from the line of stability.

\footnotetext{
${ }^{\mathrm{a}} \mathrm{e}$-mail: jsmallcombe@ triumf.ca
} 

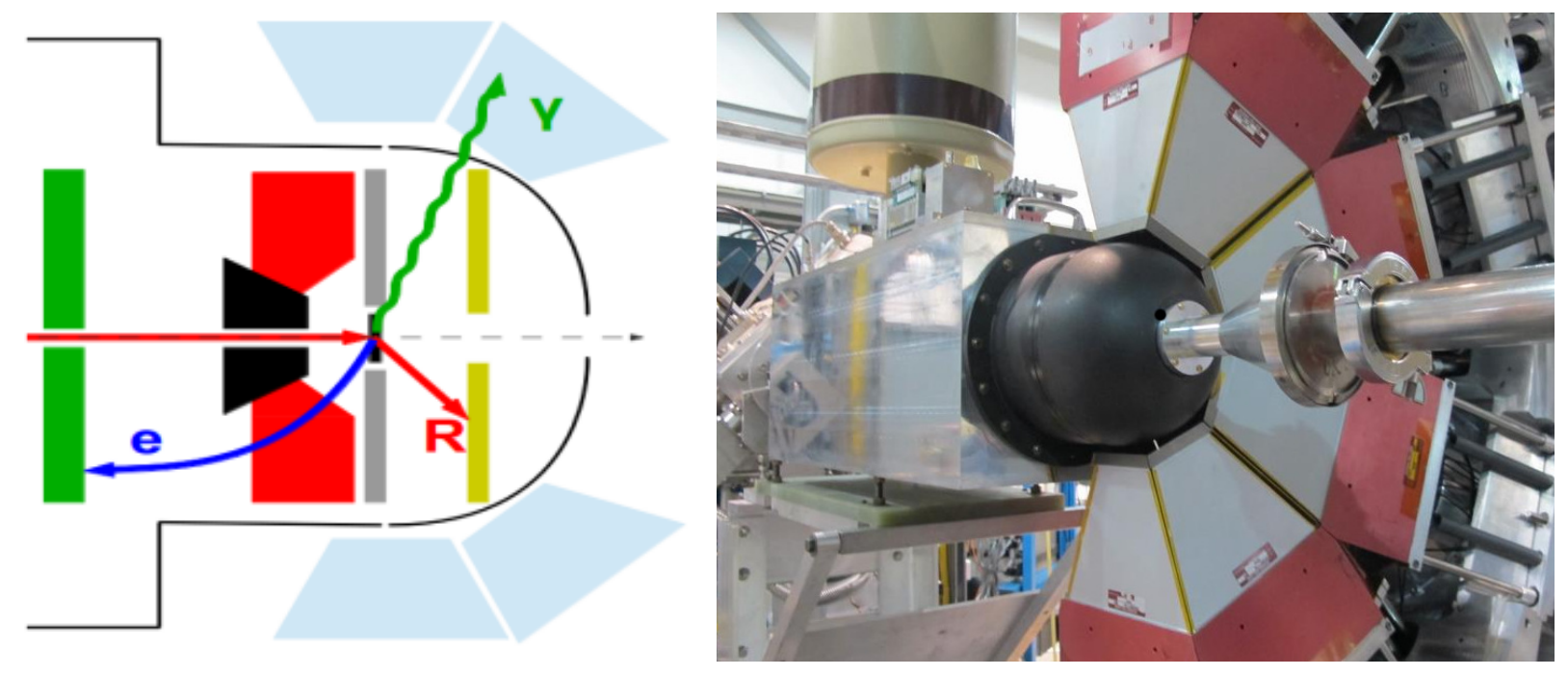

Figure 1. Left: Schematic of the primary components of SPICE as described in the text, Si(Li) detector (green), photon-shield (black), magnetic lens (red), recoil detector (yellow) and TIGRESS High-Purity Germanium (HPGe) clovers (blue). Lines show typical path of electrons (e), $\gamma$ rays $(\gamma)$, recoiling heavy ions $(\mathrm{R})$ and radioactive beam (incident from left). Right: Photograph of the SPICE chamber mounted alongside one half of the TIGRESS array.

\section{SPectrometer for Internal Conversion Electrons}

SPICE consists of an electron detector, photon shield and a magnetic lens. A schematic of the arrangement is shown in Fig. 1. Electrons are detected in an annular lithiumdrifted silicon $(\mathrm{Si}(\mathrm{Li}))$ detector of $6.1 \mathrm{~mm}$ thickess which is cooled by a liquid nitrogen coldfinger to $-100^{\circ} \mathrm{C}$. The considerable thickness of the detector allows for complete energy collection, hence increasing detection efficiency, even for highly penetrating electrons with several $\mathrm{MeV}$ of energy. Cooling allows the detector to achieve a resolution of FWHM $<5 \mathrm{keV}$; this fact is demonstrated in Fig. 2 which show the raw electron spectrum taken with a ${ }^{207} \mathrm{Bi}$ source. The detector has an outer diameter of $10 \mathrm{~cm}$ and a central hole of diameter $10 \mathrm{~mm}$, through which radioactive beams delivered from ISAC pass before striking the reaction target. The detector is segmented into 120 individual segments arranged as 12 azimuthal sectors and 10 rings. The electron detector is located $111.3 \mathrm{~mm}$ upstream of the target and is shielded from direct view of the target by a multi-layer (tungsten-tantalum alloy, tin, copper) photon shield, to block direct irradiation by X-rays and $\gamma$ rays. The magnetic lens collects and directs electrons emitted from the target position around the photon shield to the electron detector. The magnetic lens consists of four subassemblies of high grade neodymium iron boron $(\mathrm{NdFeB})$ rare-earth permanent magnet, arranged around the photon shield. The lens is oriented such that the high- $Z$ material has a minimal effect on $\gamma$-ray detection efficiency of the coupled TIGRESS array [5], as each sub-assembly is aligned with the suppression shield of the HPGe Clover detectors. Through a detailed design process [4] three magnetic lens designs have been developed (and two built) to cover the energy range from $100 \mathrm{keV}$ to $4 \mathrm{MeV}$. An absolute efficiency greater than $15 \%$ is achieved, which far

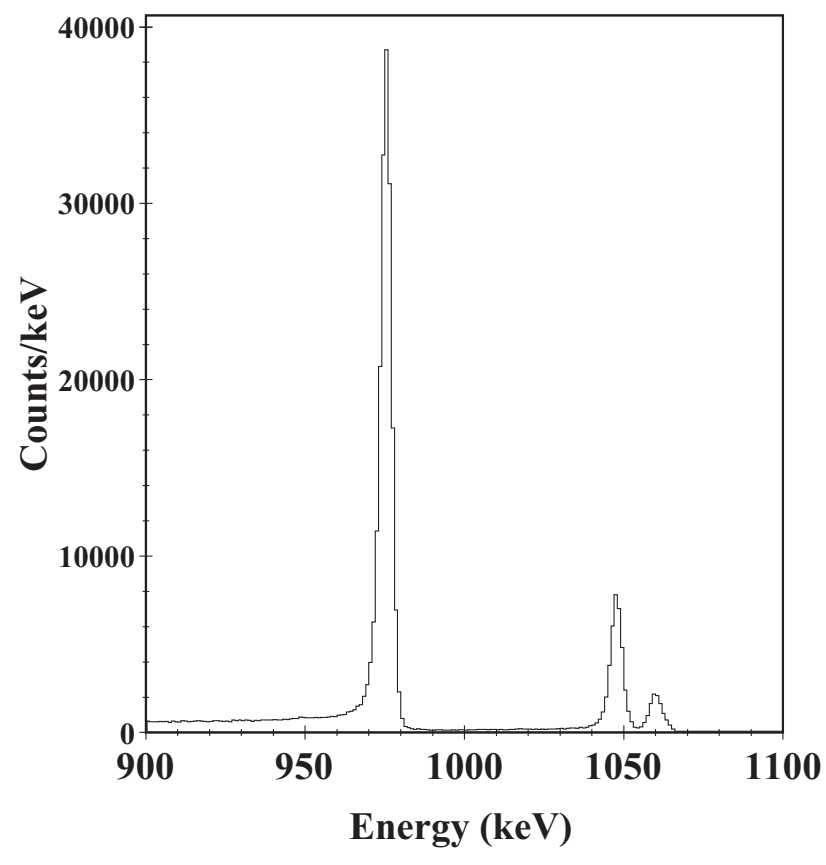

Figure 2. Raw electron spectrum in SPICE from a ${ }^{207} \mathrm{Bi}$ source, showing three distinct peaks of the $\mathrm{K}, \mathrm{L}$ and $\mathrm{M}$ electrons associated with the 1063.7-keV $\gamma$ ray. This clearly demonstrates the impressive optimum experimental resolution SPICE can achieve with FWHM $=4.5 \mathrm{keV}$ at $1 \mathrm{MeV}$.

exceeds the natural solid angle coverage of the detector. A variety of particle detectors can be coupled with SPICE in the downstream hemisphere of the target chamber to detect coincident heavy ions. An S3 type silicon CD detector has been used so far. 


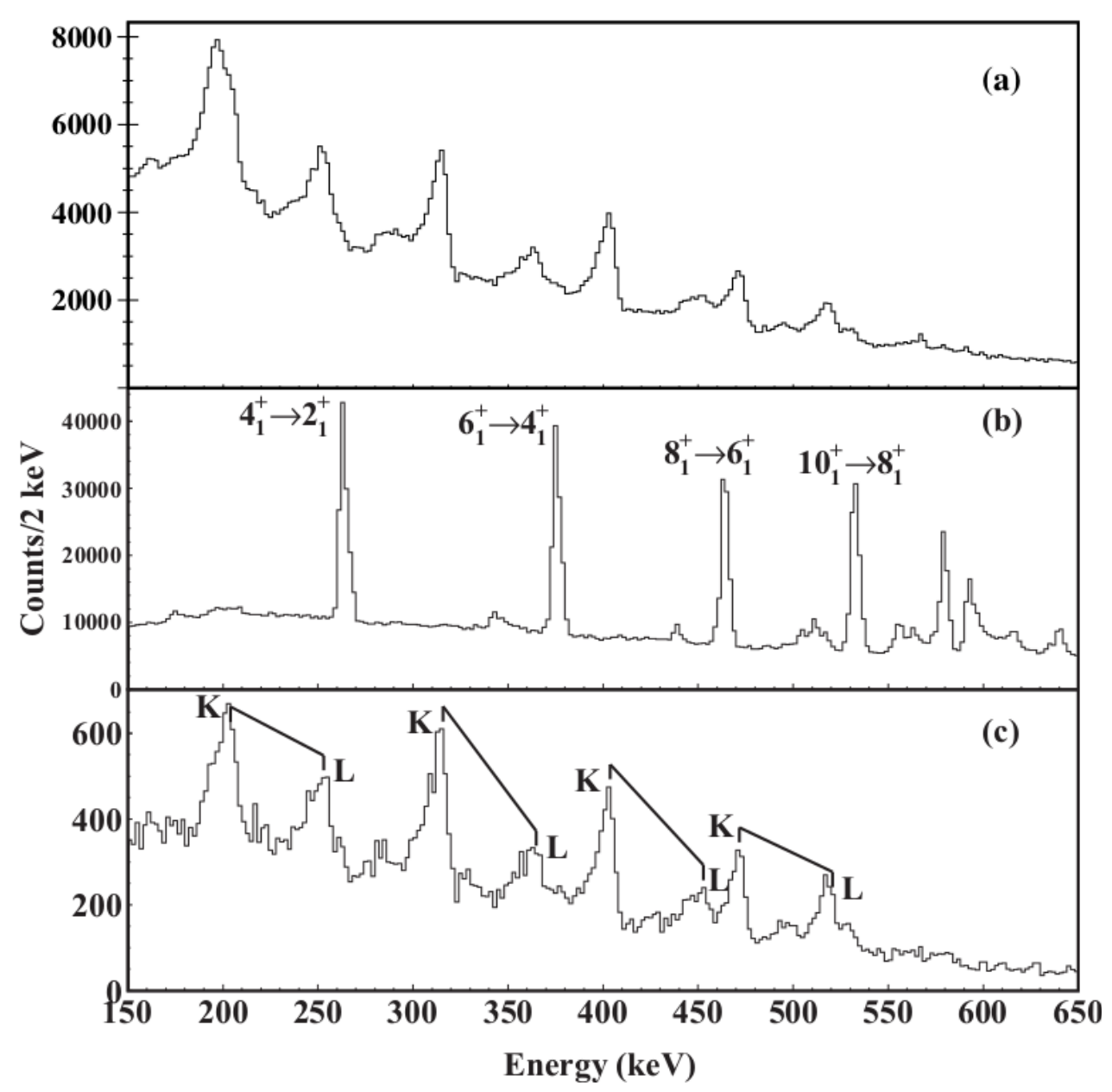

Figure 3. In-beam data from 20 hours of data for a $5.7-\mathrm{MeV} / \mathrm{u}{ }^{12} \mathrm{C}$ beam at $1 \times 10^{8} \mathrm{pps}$, impinging on a $4-\mathrm{mg} / \mathrm{cm}^{2}{ }^{152} \mathrm{Sm}$ target. The raw electron singles spectrum from SPICE (a) is dominated by transitions from the yrast band of ${ }^{160} \mathrm{Er}$ following fusion evaporation reactions. This raw singles spectrum shows that even with no coincidence requirements, SPICE still yields a formidable peak-to-total ratio for a conversion electron spectrometer. The gated $\gamma$-ray (b) and internal conversion electron (c) spectra are produced by applying a coincidence requirement with an intra-band $\gamma$-ray, within the yrast band of ${ }^{160} \mathrm{Er}$, on $\gamma-\gamma$ and electron- $\gamma$ matrices. The intra-band $\gamma$ rays, $K$ electrons (binding energy $58 \mathrm{keV}$ ) and $L$ electrons (binging energy $10 \mathrm{keV}$ ) of ${ }^{160} \mathrm{Er}$ are labeled. Peak ratios between these two spectra, combined with the detector efficiencies, can be used to produce conversion coefficient measurements.

\section{Commissioning Experiment}

A beam of ${ }^{12} \mathrm{C}$ at an energy of $5.7 \mathrm{MeV} / \mathrm{u}$ and an intensity of $10-100 \mathrm{ppA}$ (about $1 \times 10^{8}-1 \times 10^{9} \mathrm{pps}$ ) was directed at targets of ${ }^{152} \mathrm{Sm}\left(4 \mathrm{mg} / \mathrm{cm}^{2}\right)$ and ${ }^{196} \mathrm{Pt}\left(2.9 \mathrm{mg} / \mathrm{cm}^{2}\right)$. The beam energy was such that both Coulomb excitation and fusion-evaporation reactions were possible. This setup produced several data sets with different kinematics, multiplicities and target effects. The raw, un-gated, electron spectra and $\gamma$-ray spectra for each target were dominated by the fusion-evaporation channels ${ }^{152} \mathrm{Sm}\left({ }^{12} \mathrm{C}, 4 n\right){ }^{160} \mathrm{Er} *$ and ${ }^{196} \mathrm{Pt}\left({ }^{12} \mathrm{C}, 4 n\right){ }^{204} \mathrm{Po}$, respectively. The platinum target was backed by a $10-\mathrm{mg} / \mathrm{cm}^{2}$ stopper foil of natural $\mathrm{Pb}$ to catch recoiling nuclei, so that isomeric states in ${ }^{204} \mathrm{Po}$ could be studied. This will be discussed in a future publication. Figure 3 a shows the fusion-evaporation dominated spectrum for ${ }^{152} \mathrm{Sm}+{ }^{12} \mathrm{C}$; even this raw spectrum, without coincidence gating, kinematic correction or background subtraction, shows reasonable peak-to-total ratios and contains sufficient distinct peaks for good fitting. Figures $3 b$ and $3 \mathrm{c}$ demonstrate the effectiveness of $\gamma$-ray coincidence gating. The high efficiency and resolution of TIGRESS allows for clean gating of data and further the identification of and subtraction of background (not shown here). Requiring detection of a scattered ${ }^{12} \mathrm{C}$ beam ion in the downstream silicon detector, in coincidence with detected electrons and $\gamma$ rays, cleanly selects the Coulomb excitation channels ${ }^{152} \mathrm{Sm}\left({ }^{12} \mathrm{C},{ }^{12} \mathrm{C}\right){ }^{152} \mathrm{Sm} *$ and ${ }^{196} \mathrm{Pt}\left({ }^{12} \mathrm{C},{ }^{12} \mathrm{C}\right){ }^{196} \mathrm{Pt} *$. Figure 4 shows the effectiveness of a coincident heavy ion gate for the ${ }^{196} \mathrm{Pt}$ target. These spectra are gated only on a recoiling ion in the downstream silicon detector. Without further subtraction, this is sufficient to remove all fusion-evaporation channels and show only the Coulmb excitation data. By combining both heavy-ion and $\gamma$-ray coincidence gating shown here, as well as background subtraction and kinematic corrections currently being implemented, SPICE will be an extremely clean, highresolution and high-efficiency device for internal electron spectroscopy. 


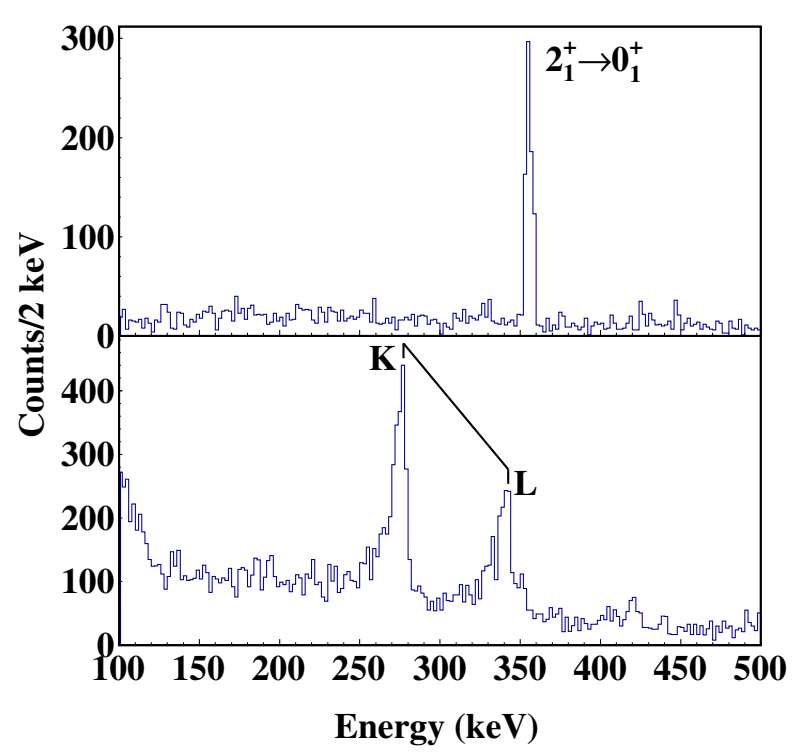

Figure 4. In-beam $\gamma$-ray (top) and internal conversion electron (bottom) data showing 2 hours of Coulomb excitation data for a $5.7-\mathrm{MeV} / \mathrm{u}{ }^{12} \mathrm{C}$ beam at $1 \times 10^{8} \mathrm{pps}$, impinging on a 2.9 $\mathrm{mg} / \mathrm{cm}^{2}{ }^{196} \mathrm{Pt}$ target, requiring a coincident heavy ion detected in the downstream recoil detector. The ${ }^{196} \mathrm{Pt} 2_{1}^{+} \rightarrow 0_{1}^{+} K$-electron and $L$-electron peaks are both prominent without background subtraction or $\gamma$-ray gating.

\section{Outlook}

The spectra presented here represent only $\sim 1 \%$ of the commissioning experiment data. Analysis of the full dataset, to demonstrate the first E0 measurement, is currently underway. The effectiveness of SPICE will be further improved by ongoing development of analysis techniques, a following publication will demonstrate the effectiveness of kinematic correction and add-back in the SPICE detector. Development of alternative downstream detectors is in progress to study states of interest populated using a variety of reaction mechanisms. The first experimental measurements with SPICE and TIGRESS are expected to take place in 2016.

\section{Acknowledgements}

The SPICE collaboration would like to thank SEMIKON Detector $\mathrm{GmbH}$, for developing the segmented $\mathrm{Si}(\mathrm{Li})$ detector. Construction of SPICE has been funded by the Canada Foundation for Innovation and the Ontario Ministry of Research and Innovation. This work was supported in part by Natural Sciences and Engineering Research Council (NSERC) of Canada. TRIUMF receives federal funding via a contribution agreement through the National Research Council of Canada.

\section{References}

[1] M. G. Mayer and J. H. D. Jensen, Elementary Theory of Nuclear Structure (Wiley, New York, 1955).

[2] S. Bohr and B. R. Mottelson, Nuclear Structure II (W. A. Benjamin, 1975).

[3] K. Heyde and J. L. Wood, Rev. Mod. Phys. 83, 1467 (2011).

[4] S. Ketelhut, L. J. Evitts, A. B. Garnsworthy, et al., Nuc. Inst. Meth. A 753, 154 (2014).

[5] G. Hackman, C. E. Svensson, Hyperfine Interactions 225, 241 (2014). 